Please cite this paper as follows:

Jeng-Tzong Chen and Hong-Ki Hong Dual Boundary Integral Equations at a Corner Using Contour Approach Around Singularity, Advances in Engineering Software, Vol.21, pp.169-178, 1994. 


\title{
Dual boundary integral equations at a corner using contour approach around singularity
}

\author{
J. T. Chen \\ Department of Harbor and River Engineering, Taiwan Ocean University, Keelung, Taiwan, Republic of China \\ $\&$ \\ H.-K. Hong \\ Department of Civil Engineering, Taiwan University, Taipei, Taiwan, Republic of China
}

(Received 17 August 1994; revised version received 17 October 1994; accepted 3 November 1994)

\begin{abstract}
A dual integral formulation for the Laplace equation problem with a corner is derived by using the contour approach surrounding the singularity. It is found that using the contour approach the jump term comes half and half from the free terms in the $L$ and $M$ kernel integrations, which is different from the limiting process from an interior point to a boundary point where the jump term comes from the $L$ kernel only. Thus, the definition of the Hadamard principal value for hypersingular integration at the collocation point of a corner is extended to a generalized sense for both the tangent and normal derivative of double layer potentials in comparison with the conventional definition. Two regularized versions of dual boundary integral equations with corners are proposed to avoid the boundary effect and are tested by an example. The numerical implementation is incorporated in the BEPO2D program. Also, a numerical example with a Dirichlet boundary condition on the corner is verified to determine the validity of the dual integral formulation.
\end{abstract}

Key words: dual boundary integral equations, dual boundary element method, corner, regularized method.

\section{INTRODUCTION}

A dual integral formulation for crack problems was developed in $1986^{1}$ and published in $1988^{2}$ and was extended to the Laplace equation with a degenerate boundary. ${ }^{3,4}$ The numerical implementation has been termed the dual boundary element method by Portela $e t$ $a l^{5}$ The formulations have been mainly applied to problems with a smooth boundary. However, a nonsmooth boundary frequently occurs in the description of many engineering problems, so the ability to handle this situation is not trivial. The nonsmooth boundary introduces a corner or edge, which makes the normal vector and normal flux at a corner undefined. How to accurately simulate the potential and potential gradient near a corner has received much attention in the boundary element method. For a corner problem, the double node technique has been discussed by Banerjee and Butterfield. ${ }^{6}$ Alarcon et al. ${ }^{7}$ used the transformation of tangent flux and normal flux to establish the constraint equation to ensure a unique solution. Walker and Fenner ${ }^{8}$ pointed out that an error will be present in computing the normal flux independently of the BEM, so they provided a nonlinear relationship for the tangent and normal fluxes. However, if the interior angle is near $90^{\circ}$, the ill-condition will occur. Therefore, the hypersingular equation has been utilized to provide a constraint at a corner in an analytical way. Gray and Manne ${ }^{9}$ have applied the hypersingular equation as an additional constraint to ensure a unique solution by a limiting process from an interior point to a corner. The three dimensional case was also extended by Gray and Lutz. ${ }^{10}$ From the viewpoint of dual integral equations, singular and hypersingular equations can provide sufficient constraints for a singular system with a corner. In the case of a nonsmooth boundary, e.g. a corner point, the jump terms of singular and hypersingular integral equations are the same in the former derivations as reported by Lutz et al. ${ }^{11}$ and Chen and Hong. ${ }^{12}$ Since the 


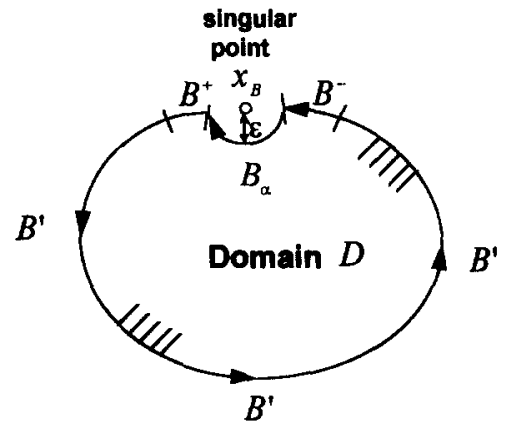

Fig. 1. The considered boundary integration path.

hypersingular integral equation can provide an additional constraint for the corner problem with Dirichlet boundary conditions, the jump term on the corner from the interior domain to the exterior domain must be examined and verified by example. This term will be corrected in this paper as the point is collocated at the corner with the normal vector before or after the corner in comparison with the results of Lutz et al. ${ }^{11}$ To derive the free terms in hypersingular equations, the bump- contour approach around the singularity is considered and is compared with the limiting process by using an analytical integral. Therefore, the dual integral equations for a corner can be derived and can be applied to solve a corner problem with Dirichlet boundary conditions by collocating the same geometry point with different normal vectors before and after the corners. Following the same symbols as in Ref. 3 of $U$, $L, T$ and $M$ kernels for a single layer kernel and its normal derivative, a double layer kernel and its normal derivative, respectively, three alternatives for constraint equations can be chosen: (1) by the $U, T$ equation and the $L^{-}, M^{-}$equation with the collocation point before the corner; (2) by the $U, T$ equation and the $L^{+}, M^{+}$ equation with the collocation point after the corner; (3) by the $L^{-}, M^{-}$equation and the $L^{+}, M^{+}$equation with the collocation point using different normal vectors before and after the corners. In order to avoid the boundary effect, two regularization techniques will be summarized. Numerical examples are shown to demonstrate the validity of the present formulations.

Table 1. Properties of difierent kinds of potentials across smooth boundary

\begin{tabular}{|c|c|c|c|c|c|c|}
\hline $\begin{array}{l}\text { Kernel } \\
\text { function } \\
K(s, x) \\
\text { direct } \\
\text { method }\end{array}$ & $U(s, x)$ & $T(s, x)$ & $L(s, x)$ & $M(s, x)$ & $L^{\mathrm{t}}(s, x)$ & $M^{\mathrm{t}}(s, x)$ \\
\hline $\begin{array}{l}\text { Kernel } \\
\text { function } \\
K(x, s) \\
\text { indirect } \\
\text { method }\end{array}$ & $U(x, s)$ & $U^{*}(x, s)$ & $T(x, s)$ & $T^{*}(x, s)$ & $T^{\mathrm{t}}(x, s)$ & $T^{* \mathrm{t}}(x, s)$ \\
\hline $\begin{array}{l}\text { Singularity } 1 D \\
\text { Singularity } 2 D \\
\text { Singularity } 3 D\end{array}$ & $\begin{array}{c}O(r) \\
O(\ln (r)) \\
O(1 / r)\end{array}$ & $\begin{array}{c}O(1) \\
O(1 / r) \\
O\left(1 / r^{2}\right)\end{array}$ & $\begin{array}{c}O(1) \\
O(1 / r) \\
O\left(1 / r^{2}\right)\end{array}$ & $\begin{array}{l}O(\delta(r)) \\
O\left(1 / r^{2}\right) \\
O\left(1 / r^{3}\right)\end{array}$ & $\begin{array}{c}O(1) \\
O(1 / r) \\
O\left(1 / r^{2}\right)\end{array}$ & $\begin{array}{l}O(\delta(r)) \\
O\left(1 / r^{2}\right) \\
O\left(1 / r^{3}\right)\end{array}$ \\
\hline $\begin{array}{l}\text { Density } \\
\text { function } \\
\mu(s)\end{array}$ & $-t$ & $u$ & $-t$ & $u$ & $-t$ & $u$ \\
\hline $\begin{array}{l}\text { Potential } \\
\text { type } \\
\int K(s, x) \mu(s) \mathrm{d} s\end{array}$ & $\begin{array}{l}\text { Single } \\
\text { layer }\end{array}$ & $\begin{array}{c}\text { Double } \\
\text { layer }\end{array}$ & $\begin{array}{c}\text { Normal } \\
\text { derivative } \\
\text { of single } \\
\text { layer } \\
\text { potential }\end{array}$ & $\begin{array}{c}\text { Normal } \\
\text { derivative } \\
\text { of double } \\
\text { layer } \\
\text { potential }\end{array}$ & $\begin{array}{l}\text { Tangent } \\
\text { derivative } \\
\text { of single } \\
\text { layer } \\
\text { potential }\end{array}$ & $\begin{array}{c}\text { Tangent } \\
\text { derivative } \\
\text { of double } \\
\text { layer } \\
\text { potential }\end{array}$ \\
\hline $\begin{array}{l}\text { Continuity } \\
\text { across } \\
\text { boundary }\end{array}$ & Continuous & Discontinuous & Discontinuous & $\begin{array}{l}\text { Pseudo- } \\
\text { continuous }\end{array}$ & Continuous & Discontinuous \\
\hline $\begin{array}{l}\text { Free term } \\
\text { method(1) }\end{array}$ & No jump & $\pi u$ & $-\pi t$ & No jump & No jump & $\pi u^{\prime}$ \\
\hline $\begin{array}{l}\text { Free term } \\
\text { method(2) }\end{array}$ & No jump & $\pi u$ & $-\frac{1}{2} \pi t$ & $-\frac{1}{2} \pi t$ & $-\frac{1}{2} \pi u^{\prime}$ & $-\frac{1}{2} \pi u^{\prime}$ \\
\hline $\begin{array}{l}\text { Jump term } \\
\operatorname{method}(1)\end{array}$ & No jump & $2 \pi u$ & $-2 \pi t$ & No jump & No jump & $2 \pi u^{\prime}$ \\
\hline $\begin{array}{l}\text { Jump term } \\
\operatorname{method}(2)\end{array}$ & No jump & $2 \pi u$ & $-\pi t$ & $-\pi t$ & $\pi u^{\prime}$ & $\pi u^{\prime}$ \\
\hline $\begin{array}{l}\text { Principal } \\
\text { value sense }\end{array}$ & $R P V$ & $C P V$ & $C P V$ & $H P V$ & $C P V$ & $H P V$ \\
\hline
\end{tabular}




\section{DUAL INTEGRAL FORMULATION OF BEM WITH A CORNER}

The dual boundary integral equations for the potential $u$ can be derived as

$$
\begin{aligned}
& 0=\int_{B_{\alpha}+B^{\prime}}\{T(s, x) u(s)-U(s, x) t(s)\} \mathrm{d} B(s) \\
& 0=\int_{B_{\alpha}+B^{\prime}}\{M(s, x) u(s)-L(s, x) t(s)\} \mathrm{d} B(s) \\
& 0=\int_{B_{\alpha}+B^{\prime}}\left\{M^{\mathrm{t}}(s, x) u(s)-L^{\mathrm{t}}(s, x) t(s)\right\} \mathrm{d} B(s)
\end{aligned}
$$

where $u(s)$ and $t(s)$ denote the potential and its normal flux on the boundary point $s$, respectively, $B^{\prime}$ and $B_{\alpha}$ are the contour integration path not including the singularity inside the domain, $D$, as shown in Fig. 1, and $U, T, L, M, L^{\mathrm{t}}$ and $M^{\mathrm{t}}$ are the six kernel functions ${ }^{4}$ in the dual integral equations with the properties shown in Table 1. The $U, M$ kernels are weakly singular and hypersingular, respectively, while the $T, L$ kernels are strongly singular. For the single and double layer kernels, Aliabadi et al. ${ }^{13,14}$ have employed a Taylor expansion to reduce the singularity order. Equations (2) and (3) are different in the direction of the derivative on the collocation point $x$. The superscript ' $t$ ' in eqn (3) denotes the tangent vector. The $B_{\alpha}$ integration path in Fig. 1 denotes the contour integration around the singularity with radius $\epsilon$, and $B^{\prime}+B^{+}+B^{-}$is just the definition of the integration region of the Cauchy principal value. The $B^{+}$and $B^{-}$denote two of the elements in the $B^{\prime}$ boundary near singularity as shown in Fig. 1. First of all, integrate the $B_{\alpha}$ path integration to obtain the free terms for the six kernel functions.

Based on Fig. 2 without loss of generality, there are the following notations:

$$
\begin{aligned}
& x=(0,0) \\
& s=(\epsilon \cos (\theta),-\epsilon \sin (\theta)) \\
& r=|x-s| \\
& y_{1}=-\epsilon \cos (\theta) \\
& y_{2}=\epsilon \sin (\theta) \\
& n(s)=\left(n_{1}, n_{2}\right)=(-\cos (\theta), \sin (\theta)) \\
& n(x)=\left(\bar{n}_{1}, \bar{n}_{2}\right)=(0,1) \text { for normal derivative } \\
& s(x)=\left(\bar{n}_{1}, \bar{n}_{2}\right)=(1,0) \text { for tangent derivative } \\
& u(s)=u(x)+\frac{\partial u}{\partial x} \epsilon \cos (\theta)-\frac{\partial u}{\partial y} \epsilon \sin (\theta) \\
& t(s)=-\frac{\partial u}{\partial x} \cos (\theta)+\frac{\partial u}{\partial y} \sin (\theta)
\end{aligned}
$$

where $s(x)$ in eqn (11) denotes the tangent vector on the point $x$ with components $(1,0)$ as shown in Fig. 2 . Since

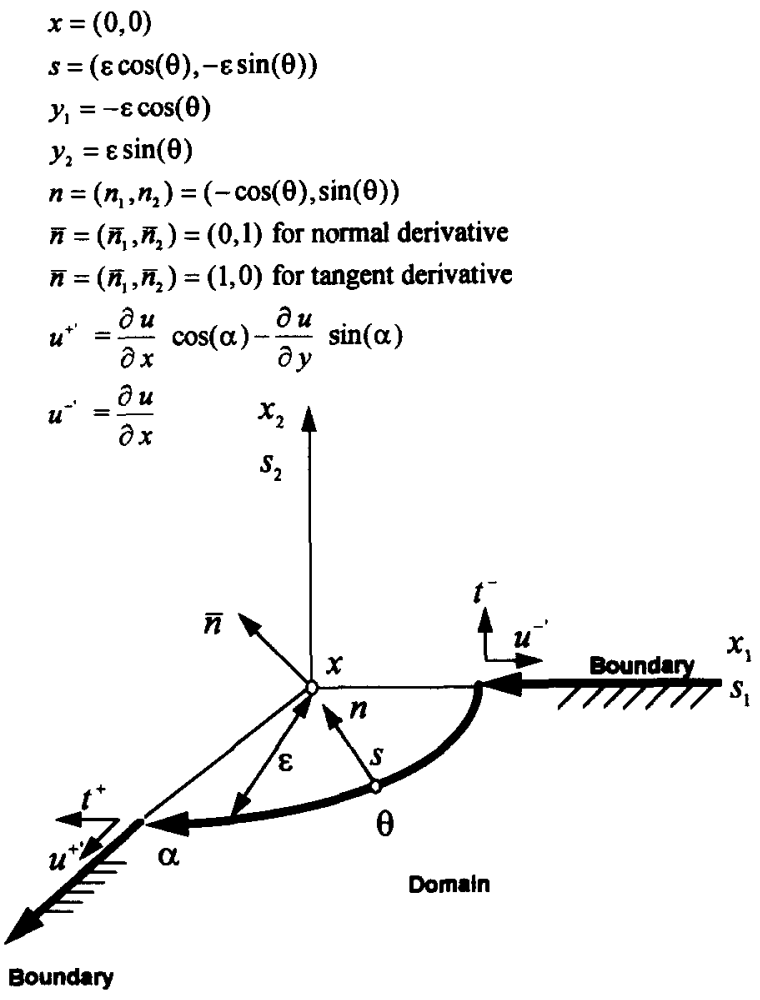

Fig. 2. Notations of the integration path around a corner.

the corner is considered with two normal vectors before and after the corners, define the following symbols:

$$
\begin{aligned}
t^{-} & =\frac{\partial u}{\partial y} \\
t^{+} & =-\frac{\partial u}{\partial x} \sin (\alpha)-\frac{\partial u}{\partial y} \cos (\alpha) \\
u^{-\prime} & =\frac{\partial u}{\partial x} \\
u^{+\prime} & =\frac{\partial u}{\partial x} \cos (\alpha)-\frac{\partial u}{\partial y} \sin (\alpha)
\end{aligned}
$$

where $\alpha$ is the interior angle of the corner, $t^{-}$and $t^{+}$are normal derivatives on the boundary point before a corner and after a corner, respectively, and $u^{-1}, u^{+\prime}$ are tangent derivatives along the boundary before and after a corner.

According to the related symbols in Fig. 3, the free terms of the six kernels will be derived as in the following.

(1) Single layer potential due to $U(s, x)=\ln (r)$ :

$$
\int_{B \alpha} U(s, x) t(s) \mathrm{d} B(s)=\epsilon \ln (\epsilon) \text { (finite value) }
$$

The free term is zero since $\epsilon \ln (\epsilon)$ approaches zero as the radius $\epsilon$ approaches zero.

(2) Double layer potential due to $T(s, x)=-y_{i} n_{i} / r^{2}$ :

$$
\int_{B \alpha} T(s, x) u(s) \mathrm{d} B(s)=-\alpha u(x)+\epsilon \text { (finite value) }
$$

As $\epsilon$ approaches zero, the free term is $-\alpha u(x)$. 
(1) $U(s, x)$

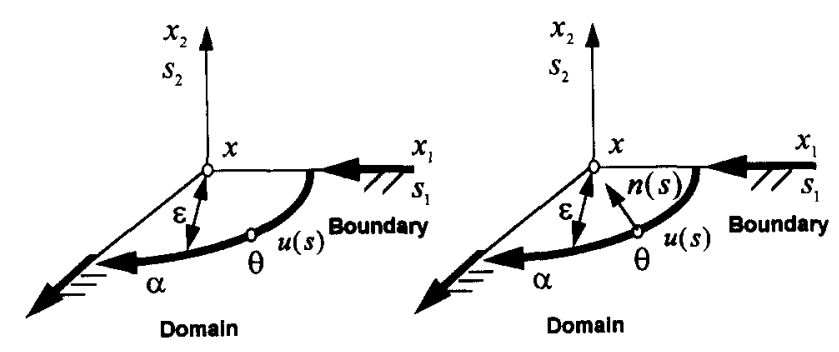

(3) $L(s, x)$

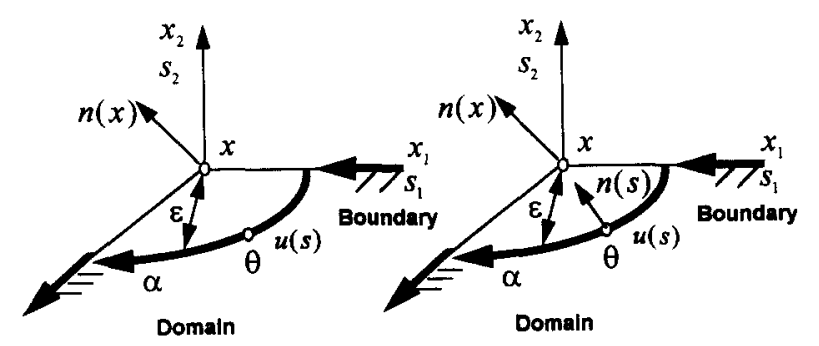

Fig. 3. Related symbols around a corner.

(3) Normal derivative of single layer potential due to $L(s, x)=y_{i} \bar{n}_{i} / r^{2}$ :

$$
\int_{B \alpha} L(s, x) t(s) \mathrm{d} B(s)=c t(x)+d u^{\prime}(x)
$$

where

$$
\begin{aligned}
& c=\frac{(-\sin (2 \alpha)+2 \alpha)}{4} \\
& d=\frac{(\cos (2 \alpha)-1)}{4}
\end{aligned}
$$

As $\epsilon$ approaches zero, the free term is $c t(x)+d u^{\prime}(x)$.

(4) Normal derivative of double layer potential due to $M(s, x)=2 y_{i} y_{j} n_{i} \bar{n}_{j} / r^{4}-n_{i} \bar{n}_{i} / r^{2}$ :

$$
\begin{aligned}
\int_{B \alpha} M(s, x) u(s) \mathrm{d} B(s)= & -c t(x)-d u^{\prime}(x) \\
& + \text { boundary term }
\end{aligned}
$$

where the boundary term $B(\epsilon)$ is

$$
B(\epsilon)=\frac{1-\cos (\alpha)}{\epsilon} u(x)
$$

It is interesting to find that the free terms from the $L$ and $M$ kernels are the same except for the minus sign. The free terms contain the boundary term, which is infinite as $\epsilon$ approaches zero. By combining with the Cauchy principal value of the $\boldsymbol{M}$ kernel integration over $\boldsymbol{B}^{\prime}$ including $\boldsymbol{B}^{+}$ and $B^{-}$as shown in Fig. 1, the finite part can be extracted, and the infinity can be cancelled out. Therefore, the Hadamard principal value in the contour integration with a corner for $M$ kernel can be defined by

$$
\begin{aligned}
& H P V \int_{B} M(s, x) u(s) \mathrm{d} B(s) \\
& =C P V \int_{B} M(s, x) u(s) \mathrm{d} B(s)+\frac{1-\cos (\alpha)}{\epsilon} u(x)
\end{aligned}
$$

(5) Tangent derivative of single layer potential due to $L^{\mathrm{t}}(s, x)=y_{i} \bar{n}_{i} / r^{2}$ :

Since the tangent derivative instead of the normal derivative is considered,

$$
\begin{aligned}
& n(x) \rightarrow s(x) \\
& \int_{B \alpha} L^{\mathrm{t}}(s, x) t(s) \mathrm{d} B(s)=c^{\prime} u^{\prime}(x)-d t(x)
\end{aligned}
$$

where

$$
c^{\prime}=\frac{(\sin (2 \alpha)+2 \alpha)}{4}
$$

As $\epsilon$ approaches zero, the free term is $c^{\prime} u^{\prime}(x)-d t(x)$.

(6) Tangent derivative of double layer potential due to $M^{\mathrm{t}}(s, x)=2 y_{i} y_{j} n_{i} \bar{n}_{j} / r^{4}-n_{i} \bar{n}_{i} / r^{2}$ :

Similar to eqn (26), only change the normal derivative to tangent derivative as

$$
\begin{aligned}
& n(x) \rightarrow s(x) \\
& \int_{B \alpha} M^{\mathrm{t}}(s, x) u(s) \mathrm{d} B(s) \\
& =-c^{\prime} u^{\prime}(x)+d t(x)+\text { boundary term }
\end{aligned}
$$

where the boundary term $B(\epsilon)$ is

$$
B(\epsilon)=-\frac{\sin (\alpha)}{\epsilon} u(x)
$$

The free terms contain the boundary term, which is infinite as $\epsilon$ approaches zero. By combining with the Cauchy principal value of the $M^{t}$ kernel integration over $\boldsymbol{B}^{\prime}$ including $\boldsymbol{B}^{+}$and $\boldsymbol{B}^{-}$, the finite part can be extracted, and the infinity can be cancelled out. Therefore, the Hadamard principal value in the contour integration with a corner for $M^{t}$ kernel can be defined by

$$
\begin{aligned}
& H P V \int_{B} M^{\mathrm{t}}(s, x) u(s) \mathrm{d} B(s) \\
& =C P V \int_{B} M^{\mathrm{t}}(s, x) u(s) \mathrm{d} B(s)-\frac{\sin (\alpha)}{\epsilon} u(x)
\end{aligned}
$$

Since the basic unknowns in the BEM are the potential and the normal derivative of potential on the boundary, the tangent derivative, $u^{\prime}$, in the present formulation would be better transformed to the combination of the normal derivative before and after the corners in Fig. 4 as follows:

$$
u^{-\prime}=\frac{-1}{\sin (\alpha)}\left[t^{+}+\cos (\alpha) t^{-}\right]
$$

Therefore, the free term of the $L$ kernel of eqn (20) can be expressed as

$$
c t^{-}(x)+d u^{\prime}(x)=\frac{1}{2} \alpha t^{-}+\frac{1}{2} \sin (\alpha) t^{+}
$$




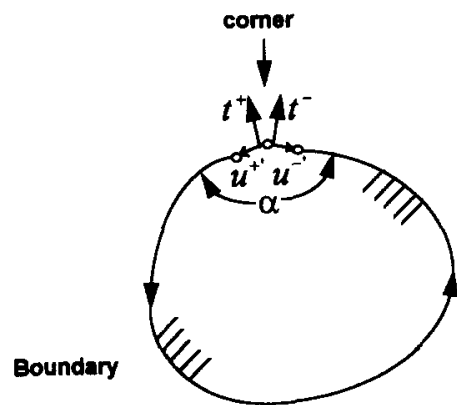

Fig. 4. Transformation of flux at a corner.

In the implementation of the BEM, it is suggested that this transformation be considered since the state variables are $t^{+}$and $t^{-}$instead of $u^{\prime}$ and $t^{-}$although $u^{\prime}$ can be expressed in terms of the numerical derivative of the nodal variables of $u$. For the free terms of the $L^{t}$ and $M^{\mathrm{t}}$ kernel, this transformation is not necessary since the tangent derivative of potential can be expressed in terms of the superposition of all the state variables, which include the potential and the normal derivative of potential on the boundary just solved by the $U, T$ and $L, M$ equations. Therefore, one can derive the following dual boundary integral equations using the transformation of eqn (32) and the expression for the tangential flux along the boundary:

$$
\begin{gathered}
\begin{aligned}
\alpha u(x)= & C P V \int_{B} T(s, x) u(s) \mathrm{d} B(s) \\
& -R P V \int_{B} U(s, x) t(s) \mathrm{d} B(s) \\
\alpha t^{-}(x)+ & \sin (\alpha) t^{+}(x)=H P V \int_{B} M(s, x) u(s) \mathrm{d} B(s) \\
& -C P V \int_{B} L(s, x) t(s) \mathrm{d} B(s) \\
2 c^{\prime} u^{\prime}(x)= & 2 d t^{-}(x)+H P V \int_{B} M^{\mathrm{t}}(s, x) u(s) \mathrm{d} B(s) \\
& -C P V \int_{B} L^{\mathrm{t}}(s, x) t(s) \mathrm{d} B(s)
\end{aligned}
\end{gathered}
$$

after using

$$
\begin{aligned}
& \int_{B^{\prime}} U(s, x) t(s) \mathrm{d} B(s)=R P V \int_{B} U(s, x) t(s) \mathrm{d} B(s) \\
& \int_{B^{\prime}} T(s, x) u(s) \mathrm{d} B(s)=C P V \int_{B} T(s, x) u(s) \mathrm{d} B(s) \\
& \int_{B^{\prime}} L(s, x) t(s) \mathrm{d} B(s)=C P V \int_{B} L(s, x) t(s) \mathrm{d} B(s)
\end{aligned}
$$

$$
\begin{aligned}
\int_{B^{\prime}} M(s, x) u(s) \mathrm{d} B(s)= & H P V \int_{B} M(s, x) u(s) \mathrm{d} B(s) \\
& -\frac{1-\cos (\alpha)}{\epsilon} u(x) \\
\int_{B^{\prime}} L^{\mathrm{t}}(s, x) t(s) \mathrm{d} B(s)= & C P V \int_{B} L(s, x) t(s) \mathrm{d} B(s) \\
\int_{B^{\prime}} M^{\mathrm{t}}(s, x) u(s) \mathrm{d} B(s)= & H P V \int_{B} M^{\mathrm{t}}(s, x) u(s) \mathrm{d} B(s) \\
& +\frac{\sin (\alpha)}{\epsilon} u(x)
\end{aligned}
$$

\section{DISCUSSIONS ON THE DUAL BOUNDARY INTEGRAL EQUATIONS AT A CORNER}

1. It is interesting to find that the hypersingular equation for the collocation point after the corner has a similar expression in comparison with the equation collocated at the point before the corner except for the change of $t^{-}$and $t^{+}$as follows:

$$
\begin{aligned}
\alpha t^{-}(x)+\sin (\alpha) t^{+}(x)= & H P V \int_{B} M^{-}(s, x) u(s) \mathrm{d} B(s) \\
& -C P V \int_{B} L^{-}(s, x) t(s) \mathrm{d} B(s) \\
\alpha t^{+}(x)+\sin (\alpha) t^{-}(x)= & H P V \int_{B} M^{+}(s, x) u(s) \mathrm{d} B(s) \\
& -C P V \int_{B} L^{+}(s, x) t(s) \mathrm{d} B(s)
\end{aligned}
$$

where $M^{-}$and $M^{+}$denote the $M$ kernels with different normal vectors collocated before and after the corner, respectively. Similarly, the same meanings of $L^{-}$and $L^{+}$ are used. Both equations can be used to solve the corner problem with Dirichlet conditions since they are linearly independent, as will be demonstrated in the following example. In the literature, the $\sin (\alpha) t^{-}(x)$ term is always omitted by Lutz et al. ${ }^{11}$ and in the book by Chen and Hong. ${ }^{12}$

2. It must be noted that the Cauchy principal value of the $L$ kernel integration at the corner, combining the Hadamard principal value of the $M$ kernel integration including the two elements of $B^{+}$and $B^{-}$in Fig. 1, exists under the request of $C^{1}$ continuity for $u$. The coefficients of $\ln (\epsilon)$ due to the $L$ and $M$ kernels can be combined and summed to zero as shown below:

$$
\left\{-\cos (\alpha) u^{-\prime}+\sin (\alpha) t^{-}+u^{+\prime}\right\} \ln (\epsilon)=0
$$

after using the definition of $u^{+\prime}$ from eqn (17). 
3. For the case of a smooth boundary, eqns (43) and (44) reduce to

$$
\begin{aligned}
\pi u(x)= & C P V \int_{B} T(s, x) u(s) \mathrm{d} B(s) \\
& -R P V \int_{B} U(s, x) t(s) \mathrm{d} B(s) \\
\pi t(x)= & H P V \int_{B} M(s, x) u(s) \mathrm{d} B(s) \\
& -C P V \int_{B} L(s, x) t(s) \mathrm{d} B(s)
\end{aligned}
$$

The representation of the tangent derivative of the boundary potential of eqn (36) is

$$
\begin{aligned}
\pi u^{\prime}(x)= & H P V \int_{B} M^{\mathrm{t}}(s, x) u(s) \mathrm{d} B(s) \\
& -C P V \int_{B} L^{\mathrm{t}}(s, x) t(s) \mathrm{d} B(s)
\end{aligned}
$$

by substituting $\alpha=\pi$. At the tip of the degenerate boundary, the dual boundary integral equations can be reduced to

$$
\begin{aligned}
0= & C P V \int_{B} T(s, x) u(s) \mathrm{d} B(s) \\
& -R P V \int_{B} U(s, x) t(s) \mathrm{d} B(s) \\
0= & H P V \int_{B} M(s, x) u(s) \mathrm{d} B(s) \\
& -C P V \int_{B} L(s, x) t(s) \mathrm{d} B(s)
\end{aligned}
$$

since $\alpha=0$. The two equations provide additional constraints for the potential at the tip on the degenerate boundary to be $u^{+}=u^{-}$.

4. For a smooth boundary, the definition of the Hadamard principal value for $M$ kernel integration is reduced to

$$
\begin{aligned}
& H P V \int_{B} M(s, x) u(s) \mathrm{d} B(s) \\
& =C P V \int_{B} M(s, x) u(s) \mathrm{d} B(s)+\frac{2}{\epsilon} u(x)
\end{aligned}
$$

One can deem the classical definition of the Hadamard principal value in the literature ${ }^{15}$ as a special case of the present formulation by setting

$$
\begin{aligned}
& M(s, x)=-\frac{1}{(x-s)^{2}} \\
& \mathrm{~d} B(s)=\mathrm{d} s
\end{aligned}
$$

Therefore, the Hadamard principal value of the $M$ kernel integration is reduced to the conventional one as follows:

$$
H P V \int_{B} \frac{-u(s)}{(x-s)^{2}} \mathrm{~d} s=C P V \int_{B} \frac{-u(s)}{(x-s)^{2}} \mathrm{~d} s+\frac{2}{\epsilon} u(x)
$$

5. If a smooth boundary is considered, the interior angle $\alpha$ is $\pi$, and the property of the free term and the jump term can be reduced to the classical potential theory as shown in Table 1. The singularity orders for the six kernel functions in one, two and three dimensional problems are shown in the third row. The seventh row shows the free term derived by method (1) shown in Fig. 5a by using a limiting process from the interior point to the boundary point using an analytical integration. ${ }^{4,17}$ The eighth row denotes the free term by contour integration around singularity by method (2) as shown in Fig. 5b. Although the final results are the same after combining the contributions from the $L$ and $M$ kernels, the intermediate free terms are different.

6. Although the boundary $B_{\alpha}$ will shrink to zero radius in the derivation, the $u(s)$ field along $B_{\alpha}$ can not be represented by $u(x)$; therefore, care should be taken in using the contour approach around the singularity in the following calculation:

$$
\begin{gathered}
\int_{B \alpha} M(s, x) u(s) \mathrm{d} B(s) \neq u(x) \int_{B \alpha} M(s, x) \mathrm{d} B(s) \\
=-u(x) C P V \int_{B} M(s, x) \mathrm{d} B(s)
\end{gathered}
$$

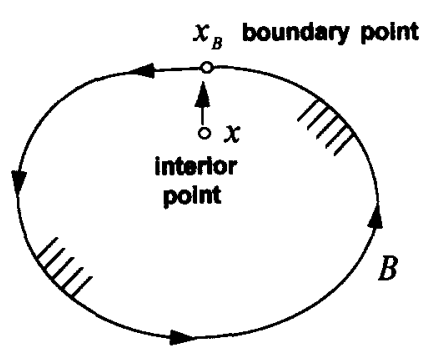

(a)

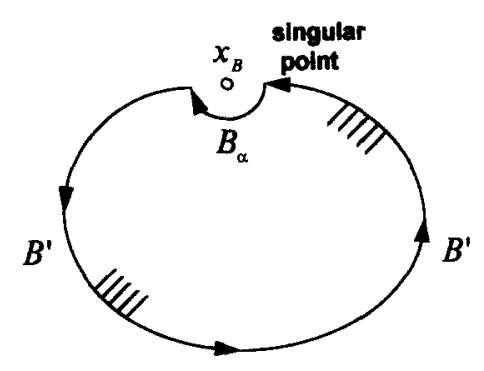

(b)

Fig. 5. (a) Limiting process from an interior point to a boundary point. (b) Contour around singularity. 
In the same way, the $t(s)$ field along $B_{\alpha}$ can not be represented by $t(x)$; so there is

$$
\int_{B \alpha} L(s, x) t(s) \mathrm{d} B(s) \neq t(x) \int_{B \alpha} L(s, x) \mathrm{d} B(s)
$$

The nonequal sign stems from the loss of free terms, $-c t(x)-d u^{\prime}(x)$. This finding will be very important to the order analysis in the following derivations for regularized versions.

\section{TWO REGULARIZED VERSIONS OF DUAL INTEGRAL EQUATIONS AT A CORNER}

Although the dual integral equations have been derived, the $C P V$ and $H P V$ concepts must be defined. In order to reduce the order of singularity, two regularized versions of dual integral equations derived by using order analysis are provided. By combining the unregularized version, three versions of dual boundary integral formulations for a boundary corner point are summarized as follows:

Version I (unregularized form):

$$
\begin{aligned}
\alpha u(x)= & C P V \int_{B} T(s, x) u(s) \mathrm{d} B(s) \\
& -R P V \int_{B} U(s, x) t(s) \mathrm{d} B(s) \\
\alpha t^{-}(x)+ & \sin (\alpha) t^{+}(x)=H P V \int_{B} M(s, x) u(s) \mathrm{d} B(s) \\
& -C P V \int_{B} L(s, x) t(s) \mathrm{d} B(s)
\end{aligned}
$$

If the potential is subtracted by the constant potential at the boundary point $x_{\mathrm{B}}$, the field of $u(x)-u\left(x_{\mathrm{B}}\right)$ also satisfies the Laplace equation, and the functional constraint is shown as follows:

Version II (regularized form with respect to $u$ ):

$$
\begin{aligned}
& 0=R P V \int_{B} T(s, x)[u(s)-u(x)] \mathrm{d} B(s) \\
& -R P V \int_{B} U(s, x) t(s) \mathrm{d} B(s) \\
& \alpha t^{-}(x)+\sin (\alpha) t^{+}(x) \\
& =C P V \int_{B} M(s, x)[u(s)-u(x)] \mathrm{d} B(s) \\
& -C P V \int_{B} L(s, x) t(s) \mathrm{d} B(s)
\end{aligned}
$$

after $x$ approaches $x_{\mathrm{B}}$ and changing $x_{\mathrm{B}}$ to $x$ again for consistency for comparison with eqns (51) and (52).

Similarly, if the potential is subtracted by the constant and linear term of potential at the boundary point $x_{B}$, the regularized solution also satisfies the Laplace equation, and the functional constraint is shown as follows:

Version III (regularized form with respect to $t$ ):

$$
\begin{aligned}
0= & R P V \int_{B} T(s, x)\left[u(s)-u(x)-u^{\prime}(x) r_{i} \bar{s}_{i}\right. \\
& \left.-t(x) r_{i} \bar{n}_{i}\right] \mathrm{d} B(s)-R P V \int_{B} U(s, x)\left[t(s)-u^{\prime}(x) n_{i} \bar{s}_{i}\right. \\
& \left.-t(x) n_{i} \bar{n}_{i}\right] \mathrm{d} B(s) \\
0= & R P V \int_{B} M(s, x)\left[u(s)-u(x)-u^{\prime}(x) r_{i} \bar{s}_{i}\right. \\
& \left.-t(x) r_{i} \bar{n}_{i}\right] \mathrm{d} B(s)-R P V \int_{B} L(s, x)\left[t(s)-u^{\prime}(x) n_{i} \bar{s}_{i}\right. \\
& \left.-t(x) n_{i} \bar{n}_{i}\right] \mathrm{d} B(s)
\end{aligned}
$$

According to the three versions of the representations, it is found that the lower the order of regularization applied, the more free terms will be present. By using eqn (49), version II can be reformulated as version I. In the three versions, no distinguishable difference is made for the boundary point and the interior point in eqns (53), (55) and (56) since only regular integration is considered. Version III is a non-singular formulation since higher order regularization is employed. Only the Gaussian quadrature rule is needed.

\section{TREATMENT OF BOUNDARY EFFECT USING REGULARIZED VERSIONS OF DUAL INTEGRAL EQUATIONS}

After obtaining all the boundary unknowns, the dual boundary integral equations for an interior point near boundary point $x_{\mathrm{B}}$ are formulated as

Version I (unregularized form):

$$
\begin{aligned}
2 \pi u(x)= & R P V \int_{B} T(s, x) u(s) \mathrm{d} B(s) \\
& -R P V \int_{B} U(s, x) t(s) \mathrm{d} B(s) \\
2 \pi t(x)= & R P V \int_{B} M(s, x) u(s) \mathrm{d} B(s) \\
& -R P V \int_{B} L(s, x) t(s) \mathrm{d} B(s)
\end{aligned}
$$

Version II (regularized form with $u$ ):

$$
\begin{aligned}
2 \pi u(x)= & R P V \int_{B} T(s, x)\left[u(s)-u\left(x_{\mathrm{B}}\right)\right] \mathrm{d} B(s) \\
& -R P V \int_{B} U(s, x) t(s) \mathrm{d} B(s)+2 \pi u\left(x_{\mathrm{B}}\right) \\
2 \pi t(x)= & R P V \int_{B} M(s, x)\left[u(s)-u\left(x_{\mathrm{B}}\right)\right] \mathrm{d} B(s) \\
& -R P V \int_{B} L(s, x) t(s) \mathrm{d} B(s)
\end{aligned}
$$


Version III (regularized form with $t$ ):

$$
\begin{aligned}
2 \pi u(x)= & R P V \int_{B} T(s, x)\left[u(s)-u\left(x_{\mathrm{B}}\right)-u^{\prime}\left(x_{\mathrm{B}}\right) r_{i} \bar{s}_{i}\right. \\
& \left.-t\left(x_{\mathrm{B}}\right) r_{i} \bar{n}_{i}\right] \mathrm{d} B(s)-R P V \int_{B} U(s, x) \\
& \times\left[t(s)-u^{\prime}\left(x_{\mathrm{B}}\right) n_{i} \bar{s}_{i}-t\left(x_{\mathrm{B}}\right) n_{i} \bar{n}_{i}\right] \mathrm{d} B(s) \\
& +2 \pi\left[u\left(x_{\mathrm{B}}\right)+u^{\prime}\left(x_{\mathrm{B}}\right) r_{i} \bar{s}_{i}+t\left(x_{\mathrm{B}}\right) r_{i} \bar{n}_{i}\right] \\
2 \pi t(x)= & R P V \int_{B} M(s, x)\left[u(s)-u\left(x_{\mathrm{B}}\right)-u^{\prime}\left(x_{\mathrm{B}}\right) r_{i} \bar{s}_{i}\right. \\
& \left.-t\left(x_{\mathrm{B}}\right) r_{i} \bar{n}_{i}\right] \mathrm{d} B(s)-R P V \int_{B} L(s, x) \\
& \times\left[t(s)-u^{\prime}\left(x_{\mathrm{B}}\right) n_{i} \bar{s}_{i}-t\left(x_{\mathrm{B}}\right) n_{i} \bar{n}_{i}\right] \mathrm{d} B(s) \\
& +2 \pi\left[u^{\prime}\left(x_{\mathrm{B}}\right) n_{i} \bar{s}_{i}+t\left(x_{\mathrm{B}}\right) n_{i} \bar{n}_{i}\right]
\end{aligned}
$$

where $r_{i}=s_{i}-\left(x_{\mathrm{B}}\right)_{i}, \bar{n}_{i}$ is the $i$ th component of the normal vector on $x_{\mathrm{B}}, \bar{s}_{i}$ is the $i$ th component of the tangent vector on $x_{\mathrm{B}}, x_{\mathrm{B}}$ is the boundary point near which the physical quantities on $x$ are to be calculated. Version I of eqns (57) and (58) often encounters the boundary effect if the quantities near the boundary are to be solved. From the computational point of view, eqn (59) is suggested for the calculation of potential near boundary point $x_{\mathrm{B}}$. The numerical experiment has been tested by Kisu and Kawahara, ${ }^{16}$ and the Gibbs phenomenon is avoided by the regularization technique. Equation (62) is recommended for the calculation of the potential gradient near the boundary point $x_{\mathrm{B}}$. A demonstrative example will be given below.

\section{ILLUSTRATIVE EXAMPLES}

In order to check the validity of the present formulation, two examples are tested in this section. The BEPO2D program in Ref. 4 is extended to solve the corner problem and include the two regularized versions of dual boundary integral equations.

\section{(1) Corner problem with Dirichlet boundary conditions as shown in Fig. 6}

Three choices of independent equations are provided as follows:

(a) By the $U, T$ equation and $L^{-}, M^{-}$equation before the corner.

(b) By the $U, T$ equation and $L^{+}, M^{+}$equation after the corner.

(c) By the $L^{-}, M^{-}$and $L^{+}, M^{+}$equation before and after the corner, respectively.

For simplicity, only three linear elements are considered. All the three versions obtain the same results for the six matrix. The matrices of $[U],[T],\left[L^{-}\right],\left[M^{-}\right],\left[L^{+}\right]$and $\left[\mathrm{M}^{+}\right]$in linear algebraic equations are shown in Fig. 6. It is found that the $[T],\left[M^{-}\right]$and $\left[M^{+}\right]$matrices pass the constant potential test since the summation of each row is zero. After substituting the given boundary conditions for $\{u\}$ as

$$
\{u\}=\left\{\begin{array}{l}
0 \\
0 \\
1 \\
1 \\
0 \\
0
\end{array}\right\}
$$

there is

$$
\{t\}=\left\{\begin{array}{r}
-\sqrt{2} \\
1 \\
1 \\
1 \\
1 \\
-\sqrt{2}
\end{array}\right\}
$$

All the three results by methods (a), (b) and (c) match the exact solution very well for the three alternative methods. According to all the known boundary data, the interior potential and potential gradient can be exactly obtained.

\section{(2) Treatment of boundary effect using the regularized version of the dual integral equations}

In order to demonstrate the boundary effect, the example with an exact solution, $u(x, y)=0.5(x-y)$, in Fig. 7 is tested. Altogether 20 elements are considered in the BEM model. The result using the unregularized version is shown in Fig. 8. The more the deterioration result is present, the nearer the point approaches to the boundary. After using the regularized version, the boundary effect can be removed. It is found that the results for the potential gradient near the boundary approaches half the data on the boundary as Kisu and Kawahara ${ }^{16}$ proposed for the potential near boundary. This tendency can be understood from the abrupt change from $2 \pi u$ or $2 \pi t$ to $\pi u$ or $\pi t$ in version I of the dual boundary integral equations from the domain point to the boundary point, respectively. By using versions II and III, the abrupt change is zero since $x$ approaches $x_{\mathrm{B}}$ so that the behavior across the boundary is continuous. Using these two formulae, one can avoid the boundary effect since the jump term is approximated to zero due to the density function near zero, and the Gibbs phenomenon can be avoided. Therefore, the physical quantities near the boundary can be accurately calculated.

\section{CONCLUSIONS}

Three versions of dual boundary integral equations at a 


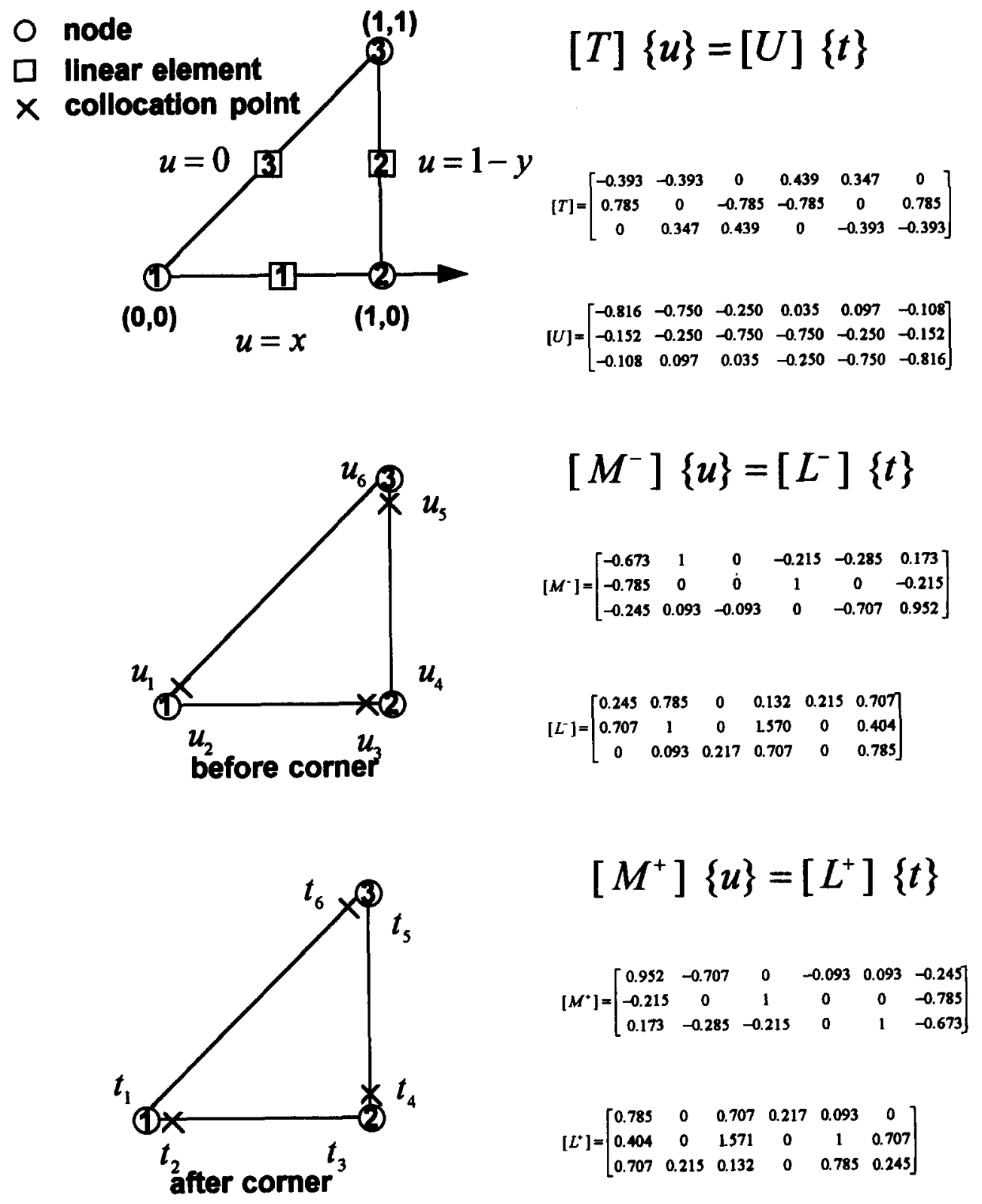

Fig. 6. Corner problem with the Dirichlet boundary conditions.

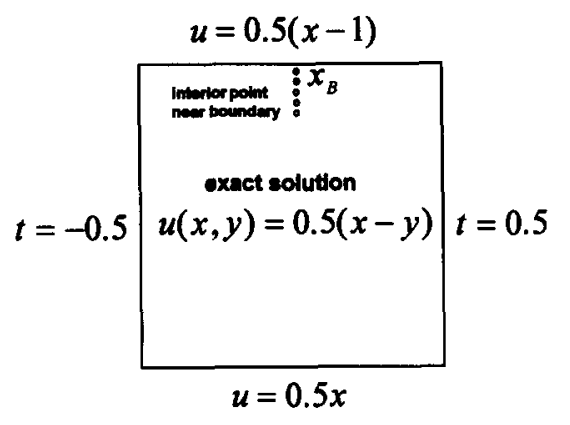

Fig. 7. Illustrative example for the boundary effect.

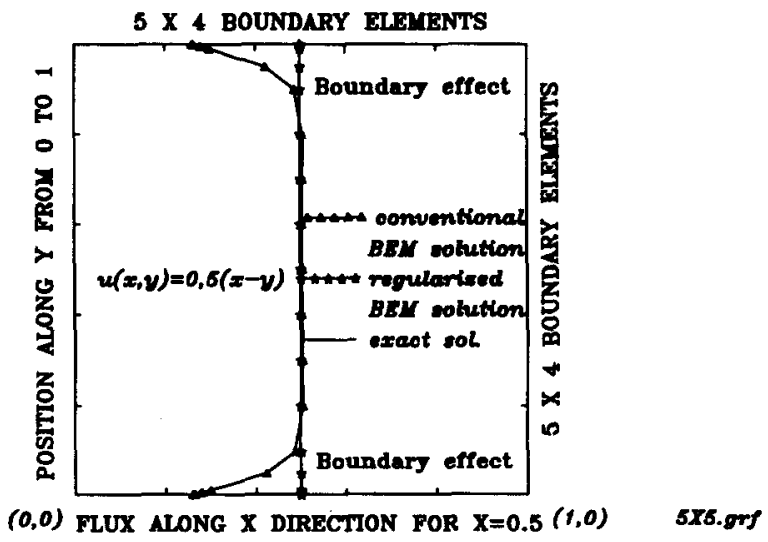

Fig. 8. Regularized solution for the potential gradient near the boundary. 
corner have been derived and implemented into the BEPO2D program. In the first version, the $H P V$ is extended to a more general definition and can be reduced back to the conventional one. In the second version, a nonhypersingular formulation is derived. The third version provides a regular formulation. It is found that the lower the order of regularization applied, the more free terms will be present. The boundary effect of the potential and potential gradient can be avoided by using versions II and III, respectively. The formulations have been tested in two examples, and the results are satisfactory.

\section{REFERENCES}

1. Chen, J. T., On Hadamard principal value and boundary integral formulation of fracture mechanics. Master thesis, under the supervision of Professor Hong-Ki Hong, Institute of Applied Mechanics, National Taiwan University, 1986.

2. Hong, H.-K. \& Chen, J. T., Derivation of integral equations in elasticity. J. Engng Mech. Div., ASCE, 114(6), Em5 (1988) 1028-44.

3. Chen, J. T. \& Hong, H.-K., On the dual integral representation of boundary value problem in Laplace equation. Bound. Elem. Abstr., 4(3) (1993) 114-16.

4. Chen, J. T., Hong, H.-K. \& Chyuan, S. W., Boundary element analysis and design in seepage flow problems with sheetpiles. Finite Elem. Anal. Design, 17 (1994) 1-20.

5. Portela, A., Aliabadi, M. H. \& Rooke, D. P., The dual boundary element method: effective implementation for crack problems. Int. J. Numer. Meth. Engng, 33 (1992) 1269-87.

6. Banerjee, P. K. \& Butterfield, R., Boundary Element Methods in Engineering Science. McGraw-Hill, London, 1981.
7. Alarcon, E., Martin, A. \& Paris, F., Boundary elements in potential and elasticity theory. Comput. Struct., 10 (1979) 351-62.

8. Walker, S. P. \& Fenner, R. T., Treatment of corners in BIE analysis of potential problems. Int. J. Numer. Meth. Engng, 28 (1989) 2569-81.

9. Gray, L. J. \& Manne, L. L., Hypersingular integrals at a corner. Engng Anal. Bound. Elem., 11 (1993) $327-34$.

10. Gray, L. J. \& Lutz, E., On the treatment of corners in the boundary element method. J. Comp. Appl. Math., 32 (1990) 369-86.

11. Lutz, E., Gray, L. J. \& Ingraffea, A. R., An overview of integration methods for hypersingular boundary integrals. Proc. BEM13 Conference. Computational Mechanics Publications, Southampton, 1991.

12. Chen, J. T. \& Hong, H.-K., Boundary Element Method, 2nd edn. New World Press, Taipei, Taiwan, 1992 (in Chinese).

13. Aliabadi, M. H., Hall, W. S. \& Phemister, T. G., Taylor expansions in the boundary element methods for Neumann problems. In Boundary Elements VII, ed. C. A. Brebbia \& G. Maier. 12.31-12.39, Computational Mechanics Publications, Southampton, 1985, pp. 12.3112.39 .

14. Aliabadi, M. H., Hall, W. S. \& Phemister, T. G., Taylor expansions for singular kernels in the boundary element method. Int. J. Numer. Meth. Engng, 21 (1985) 2221-36.

15. Tuck, E. O., Application and solution of Cauchy singular integral equations. In The Application and Numerical Solution of Integral Equations, ed. R. S. Anderson et al. Sijthoff and Noordhoff, 1980.

16. Kisu, H. \& Kawahara, T., Boundary element analysis system based on a formulation with relative quantity. Proc. BEM10 Conference. Computational Mechanics Publications, Southampton, 1988.

17. Günter, N. M., Potential Theory and Its Applications to Basic Problems of Mathematical Physics. Frederick Ungar Publishing, Co., New York, 1967. 